\title{
Macrofauna Edáfica como Indicadora em Revegetação com Leguminosas Arbóreas
}

\author{
Miriam de Oliveira Bianchi ${ }^{1}$, Rafael Nogueira Scoriza ${ }^{1}$, \\ Alexander Silva de Resende ${ }^{2}$, Eduardo Francia Carneiro Campello², \\ Maria Elizabeth Fernandes Correia ${ }^{3}$, Eliane Maria Ribeiro da Silva ${ }^{4}$ \\ ${ }^{1}$ Departamento de Solos, Universidade Federal Rural do Rio de Janeiro - UFRRJ, Seropédica/RJ, Brasil \\ ${ }^{2}$ Laboratório Leguminosas Florestais, Empresa Brasileira de Pesquisa Agropecuária - \\ Embrapa Agrobiologia, Seropédica/RJ, Brasil \\ ${ }^{3}$ Laboratório de Fauna do Solo, Empresa Brasileira de Pesquisa Agropecuária - \\ Embrapa Agrobiologia, Seropédica/RJ, Brasil \\ ${ }^{4}$ Laboratório de Micorrizas, Empresa Brasileira de Pesquisa Agropecuária - Embrapa Agrobiologia, Seropédica/RJ, Brasil
}

\begin{abstract}
RESUMO
O trabalho foi desenvolvido em uma área de pasto abandonada revegetada com leguminosas arbóreas. Localizada no município de Valença, RJ, nela foram utilizadas diferentes proporções de leguminosas arbóreas fixadoras de nitrogênio: 0\% (0L), 25\% (25L), 50\% (50L) e 75\% (75L). Para avaliação da macrofauna do solo, as amostragens foram feitas nas épocas seca e úmida, utilizando-se o método TSBF. Outras amostragens foram realizadas, ainda, em uma floresta secundária (FS) e em duas áreas de pasto (PA e PB). O processo de revegetação aumentou a diversidade da fauna do solo de uma forma geral. Formicidae foi o grupo da macrofauna de maior abundância em todas as áreas, para as duas épocas de avaliação. Na época chuvosa, houve aumento considerável na quantidade de invertebrados na camada serapilheira nos tratamentos 0L, 25L, 50L, 75L e Floresta Secundária, o que no período seco aconteceu na camada 0-10 cm.
\end{abstract}

Palavras-chave: invertebrados do solo, indicadores biológicos, qualidade do solo.

\section{Soil Macrofauna as Indicators in Tree Legume Revegetation}

\begin{abstract}
The study was conducted in an area of abandoned pasture located in the city of Valença (RJ) revegetated with tree legumes. Different proportions of nitrogen-fixing leguminous trees were used: $0 \%(0 \mathrm{~L}), 25 \%(25 \mathrm{~L}), 50 \%(50 \mathrm{~L})$ and $75 \%(75 \mathrm{~L})$. For evaluation of soil biota, samplings were made in the end of the dry season and in the rainy season. Samples were collected in the abandoned pasture, in a secondary forest, and in two grassland areas (PA and PB). The process of reforestation has increased the overall diversity of soil fauna. Formicidae was the group that stood out, being abundant in all treatments and in the two periods of collection. During the rainy season, a considerable increase in the amount of invertebrates in the litter layer was observed in the treatments $0 \mathrm{~L}, 25 \mathrm{~L}, 50 \mathrm{~L}, 75 \mathrm{~L}$ and in the secondary forest, while during the dry season, such increase occurred in the $0-10 \mathrm{~cm}$ layer.
\end{abstract}

Keywords: soil invertebrates, biological indicators, soil quality. 


\section{INTRODUÇÃO E OBJETIVOS}

Qualquer ação para restaurar um ecossistema florestal deve ser tomada no sentido de acelerar a sucessão, propiciando o aumento da biodiversidade (Moraes et al., 2013). Com esse objetivo, a arborização de pastagens, em particular com leguminosas arbóreas fixadoras de $\mathrm{N}_{2}$, visa minimizar os impactos ambientais da pecuária e incrementar a qualidade do solo (Dias et al., 2006). Esse grupo vegetal influencia e é influenciado pelas propriedades do solo e pela produtividade do ecossistema (Gei \& Powers, 2013; Binkley \& Fisher, 2013), pois contribui na manutenção dos diferentes serviços ambientais providos pela serrapilheira e pela matéria orgânica do solo e promove as interações ecológicas do ecossistema (Duarte et al., 2013).

Como a serapilheira forma uma parte substancial na cadeia trófica do solo, qualquer processo que modifique a sua taxa de aporte e qualidade, como mudanças na composição de espécies arbóreas, causa alterações na temperatura, na umidade, na incidência de luminosidade e nas condições físico-químicas do solo e, potencialmente, influencia diretamente riqueza e densidades de espécies da comunidade macrofauna (Riutta et al., 2012; Ashford et al., 2013; Moraes et al., 2013).

A macrofauna do solo, representada por um grupo diverso tanto em termos morfológicos quanto comportamentais, inclui minhocas, térmitas, formigas, diplópodes, entre outros com diâmetro corporal superior a $2 \mathrm{~mm}$ (Lavelle, 1997; Vasconcellos et al., 2013). São componentes-chave da cadeia alimentar do solo, desempenhando serviços ecossistêmicos de suporte e regulação (Jeffery et al., 2010), como: criação de macroporos que influenciam na taxa de infiltração de água e nas trocas gasosas; participam também da formação do solo e da ciclagem de nutrientes; e aumentam a resiliência do sistema e a fragmentação dos tecidos vegetais, que serão humificados por microorganismos, aumentando sua taxa de decomposição (Lepsch, 2011; Rousseau et al., 2012; Binkley \& Fisher, 2013; Collison et al., 2013; Vasconcellos et al., 2013).

Neste trabalho buscou-se avaliar a resposta da macrofauna edáfica às alterações promovidas no sistema solo-serapilheira decorrentes do processo de recuperação de uma área de pasto abandonado, na qual foi realizado plantio de leguminosas arbóreas fixadoras de nitrogênio, em diferentes proporções.

\section{MATERIAL E MÉTODOS}

O trabalho foi realizado na Fazenda Santo Antônio da Aliança, localizada nos municípios de Valença e Barra do Piraí, Estado do Rio de Janeiro, entre as coordenadas geográficas $22^{\circ} 22^{\prime} 22^{\prime \prime}$ de latitude sul e $43^{\circ} 47^{\prime} 23^{\prime \prime}$ de longitude oeste e altitude de cerca de $650 \mathrm{~m}$, em área de relevo ondulado. Denomina-se a vegetação da região fitoecológica Floresta Estacional Semidecidual (IBGE, 2012). O tipo de solo predominante é Latossolo Vermelho-Amarelo álico A moderado, com textura argilosa. O clima da região classifica-se, segundo Koppen, como Cwa, tropical de altitude, com precipitação média de $1.225 \mathrm{~mm}$ (média dos últimos 10 anos).

$\mathrm{Na}$ área de declive acentuado caracterizada como pasto abandonado foi realizado no ano de $2001 \mathrm{o}$ plantio das espécies arbóreas secundárias de valor econômico nativas da Mata Atlântica: Farinha seca [Peltophorum dubium (Spreng.) Taub.], Paineira (Ceiba speciosa A. St.-Hil.), Pau-jacaré [Piptadenia gonoacantha (Mart.) Macbr.], Jaracandá da Bahia (Dalbergia nigra Vellozo), Ipê rosa [Tabebuia heptaphylla (Vellozo) Toledo], Ipê amarelo [Tabebuia chrysotricha (Mart. ex. DC.) Standal], Sobrasil (Colubrina glandulosa Perk.), Guapuruvu [Schizolobium parahyba (Vell.) Blake], Aroeira [Lithraea molleoides (Vell.) Engl.], Cedro caia (Cedrela odorata L.), Jacarandá mimoso (Jacaranda mimosaefolia D. Don.) e Sibipiruna (Caesalpinia peltophoroides Benth.). Em consórcio, utilizaram-se quatro proporções de espécies da família Leguminosae-Mimosoideae fixadoras de nitrogênio e de rápido crescimento, como Acacia auriculada (Acacia auriculiformis A. Cunn. ex Benth.), Mangium (Acacia mangium Willd.), Sabiá (Mimosa caesalpiniifolia Benth.), Orelha de negro [Enterolobium contortisiliquum (Vell.) Morong] e Guachapele [Pseudosamanea guachapele (Kunth e Harms.)].

Formou-se um ensaio de blocos ao acaso com quatro tratamentos e quatro repetições, com as proporções de leguminosas distribuídas em 0\%, 25\%, 50\% e 75\% do total das 200 plantas utilizadas por parcela de 35 x $25 \mathrm{~m}$. O espaçamento utilizado foi de $2 \mathrm{~m}$ x $2 \mathrm{~m}$, plantadas em curva de nível. Para efeito de avaliação, os tratamentos referentes à área revegetada receberam siglas (0L, 25L, 50L, 75L) para facilitar a visualização 
e compreensão, de acordo com o percentual de leguminosas presentes em cada parcela.

Além da área revegetada foi avaliada uma mata (MA) próxima ao experimento e representativa do local de estudo, com aproximadamente 80 anos de desenvolvimento após a perda da cobertura original para o cultivo do café, caracterizada em estágio médio e avançado de regeneração, na qual a família Leguminosae é a mais representativa, principalmente pelas espécies Anadenanthera colubrina (Vell.) Brenan e Piptadenia gonoacantha (Mart.) J. F, encontrando-se alguns indivíduos do estrato superior mais proeminentes e estratificação do dossel pouco definida, o que dificultou estabelecer o número e altura dos estratos (Spolidoro, 2001). Outras duas áreas de pasto foram avaliadas, um sem a presença de animais (PB), com vegetação de gramíneas espontâneas e alguns subarbustos, e um periodicamente frequentado por animais (PA), com vegetação predominante de gramíneas do gênero Brachiaria.

As amostragens da macrofauna do solo foram realizadas ao final das épocas seca (agosto/2007) e úmida (fevereiro/2008), através do método de monolitos de solo (Anderson \& Ingram, 1993), no qual, com o auxílio de uma sonda quadrada de $0,25 \mathrm{~m} \times 0,25 \mathrm{~m}$, os blocos de solo foram retirados nas profundidades de 0-10 cm, $10-20 \mathrm{~cm}$ e $20-30 \mathrm{~cm}$, além da serapilheira superficial. Na área enriquecida com leguminosas foram coletados dois pontos de amostragem por parcela. Já para a FS e para os dois pastos foram coletados oito pontos distribuídos ao longo da área, sendo que nos dois últimos não houve coleta de serapilheira.

No laboratório, os invertebrados foram identificados em nível de grandes grupos taxonômicos como ordem, classe ou família, de acordo com Dindal (1990). Para a avaliação da comunidade da macrofauna do solo, as densidades dos diferentes grupos e do total de indivíduos por área e época foram estimadas, extrapolando-se as médias para indivíduos. $\mathrm{m}^{-2}$, associadas aos respectivos erros padrões. As medidas de diversidade também foram calculadas por área e época, como riqueza total, riqueza média, índice de diversidade de Shannon e de equitabilidade de Pielou.

Os dados coletados foram testados quanto à homogeneidade (Cochran e Barttlet, 5\%) e normalidade (Lilliefors, 5\%) no programa Saeg. Em seguida foi feita uma análise de variância, os testes de média (teste de Scott-Knott a 5\%) e análise de regressão linear no programa Sisvar. Essas análises foram utilizadas para avaliar somente os resultados obtidos na área revegetada. Para efeito de comparação entre a área revegetada e os demais tratamentos, fez-se uso do teste não paramétrico de Kruskal-Wallis no programa Saeg. Para a averiguação das diferenças na distribuição dos invertebrados no perfil do solo, dentro de cada área e para cada época, também foi realizado esse mesmo teste não paramétrico.

Com o objetivo de integrar as informações dos diferentes grupos da fauna de solo com o ambiente, foi realizada a análise de componentes principais, com o auxílio do programa Canoco, sendo reunidos os grupos em percentual inferior a $1 \%$.

\section{RESULTADOS E DISCUSSÃO}

A densidade de indivíduos da macrofauna do solo coletada não apresentou diferenças entre os tratamentos e entre as épocas de avaliação (Tabela 1), embora os tratamentos PA, PB e 50L, na época seca, e PA, 0L e FS, na época úmida, tenham apresentado os maiores valores.

Manhães et al. (2013), avaliando a fauna do solo em plantios de leguminosas arbóreas sobre pastagem também não encontrou diferenças na densidade de indivíduos associados à serapilheira entre as épocas seca e úmida do ano, porém verificou diferenças entre os locais avaliados. Essa ausência de diferenças pode estar associada ao grande domínio do grupo formicidae que, na época seca, chegou a representar $82 \%$ do número de indivíduos da fauna do solo. Vasconcellos et al. (2013) associaram a alta abundância de formigas no período seco à maior disponibilidade e menor competição por alimento, pois segundo Collison et al. (2013), nessas condições a funcionalidade dos macroinvertebrados pode ser reduzida e ocorre uma mudança na comunidade para espécies mais bem adaptadas a baixa umidade.

Essa predominância do grupo formicidae causou as diferenças nos índices de diversidade de Shannon e de equitabilidade de Pielou, principalmente no tratamento 50L (Tabela 1). A relação entre densidade de indivíduos e riqueza de grupos expressa por esses índices evidencia que na época úmida as áreas estavam representadas por uma comunidade mais diversa, na 
Tabela 1. Densidade de invertebrados e índices ecológicos da macrofauna do solo em cada área avaliada nas duas épocas de coleta.

Table 1. Densities of invertebrates and ecological indices of soil macrofauna in each area assessed in both harvests.

\begin{tabular}{ccccccc}
$*$ & Indivíduo.m & Erro padrão & Riqueza total & $\begin{array}{c}\text { Riqueza } \\
\text { média }\end{array}$ & Shannon & Pielou \\
\cline { 2 - 7 } & & \multicolumn{2}{c}{ Época seca } \\
\hline PA & $2822^{\mathrm{A}}$ & 596 & 21 & $9,13^{\mathrm{A}}$ & 1,97 & 0,45 \\
\hline PB & $2826^{\mathrm{A}}$ & 781 & 17 & $9,13^{\mathrm{A}}$ & 1,83 & 0,45 \\
\hline $0 \mathrm{~L}$ & $1580^{\mathrm{aA}}$ & 396 & 19 & $12,25^{\mathrm{bA}}$ & 1,54 & 0,36 \\
\hline $25 \mathrm{~L}$ & $1132^{\mathrm{aA}}$ & 209 & 20 & $13,00^{\mathrm{aA}}$ & 2,61 & 0,60 \\
\hline $50 \mathrm{~L}$ & $2992^{\mathrm{aA}}$ & 1111 & 18 & $11,5^{\mathrm{bA}}$ & 1,21 & 0,29 \\
\hline $75 \mathrm{~L}$ & $1322^{\mathrm{aA}}$ & 329 & 20 & $13,5^{\mathrm{aA}}$ & 2,52 & 0,58 \\
\hline FS & $2196^{\mathrm{A}}$ & 511 & 22 & $12,13^{\mathrm{A}}$ & 2,26 & 0,51 \\
\hline PA & $1802^{\mathrm{A}}$ & 573 & 23 & $10,13^{\mathrm{AB}}$ & 2,46 & 0,54 \\
\hline PB & $706^{\mathrm{A}}$ & 186 & 16 & $6,25^{\mathrm{B}}$ & 2,79 & 0,70 \\
\hline $0 \mathrm{~L}$ & $1686^{\mathrm{aA}}$ & 286 & 21 & $13,88^{\mathrm{aA}}$ & 3,27 & 0,74 \\
\hline $25 \mathrm{~L}$ & $1170^{\mathrm{aA}}$ & 225 & 21 & $13,00^{\mathrm{aA}}$ & 3,31 & 0,75 \\
\hline $50 \mathrm{~L}$ & $1164^{\mathrm{aA}}$ & 143 & 23 & $14,00^{\mathrm{aA}}$ & 3,46 & 0,77 \\
\hline $75 \mathrm{~L}$ & $1360^{\mathrm{aA}}$ & 337 & 23 & $12,88^{\mathrm{bAB}}$ & 3,06 & 0,68 \\
\hline FS & $162^{\mathrm{A}}$ & 353 & 24 & $14,25^{\mathrm{A}}$ & 2,80 & 0,61 \\
\hline
\end{tabular}

Valores com letras minúsculas (comparação entre épocas) e maísculas (comparação entre tratamentos) iguais não diferem pelos testes de Scoot-Knott a 5\% e de Kruskal-Wallis a 5\%. PA - pasto A; PB - pasto B; 0L - 0\% de leguminosas; 25L - 25\% de leguminosas; 50L - 50\% de leguminosas; 75L - 75\% de leguminosas; FS - Floresta secundária.

qual os grupos ocorreram de forma mais representativa e melhor distribuída, embora isso não signifique uma maior abundância total. Segundo Manhães et al. (2013), variações sazonais influenciam a ocorrência de grupos da fauna do solo, principalmente predadores e herbívoros, o que afeta a diversidade e equitabilidade da comunidade.

$\mathrm{Na}$ área de floresta foi observado um maior valor de riqueza total de grupos da fauna para as duas épocas. Em relação à riqueza média, segundo a análise de variância não paramétrica, somente no período chuvoso houve diferença significativa entre os tratamentos, destacando-se mais claramente diferenças de diversidade entre áreas florestadas e pasto (Tabela 1).

Analisando-se a distribuição vertical da fauna dos compartimentos do solo (Figura 1), na época seca, a maior densidade percentual foi encontrada na camada mais superficial do solo $(0-10 \mathrm{~cm})$, em todos os tratamentos, com destaque para FS e 50L. Já na época úmida houve maior homogeneidade entre a serapilheira e a camada mais superficial do solo nas áreas florestais.

Segundo Lepsch (2011), encontram-se os artrópodes do solo em grande número principalmente nos horizontes $\mathrm{O}$ e $\mathrm{A}$. O número de indivíduos presentes na profundidade 10-20 cm mostrou-se aproximadamente similar para todos os tratamentos, enquanto que em 20-30 cm a maior densidade se deu no tratamento 75L. Já no período chuvoso (Figura 1), parte considerável dos organismos foi encontrada na serapilheira, principalmente na FS, onde esse valor alcança $70 \%$ da densidade total.

Através da análise de regressão, para avaliar o padrão de distribuição dos grupos da fauna entre as diferentes proporções de leguminosas (0L, 25L, 50L e 75L), dentro de cada época, o grupo coleoptera $\left(R^{2}=92,9 ; p<0,05\right)$ apresentou uma relação positiva com o aumento percentual de leguminosas arbóreas implantadas. Segundo Dias et al. (2006), a presença de leguminosas arbóreas contribui para o aumento da densidade, riqueza e diversidade da fauna do solo, principalmente dos grupos coleoptera, araneae e formicidae. Além disto, a movimentação vertical dos coleopteros e outros organismos da serapilheira como isopoda, coleoptera, blattaria, chilopoda, opilionidae e diplopoda, está associada às mudanças de temperatura do solo que, por sua vez, é influenciada pela presença de diferentes tipos de vegetais (Rousseau et al., 2012). 

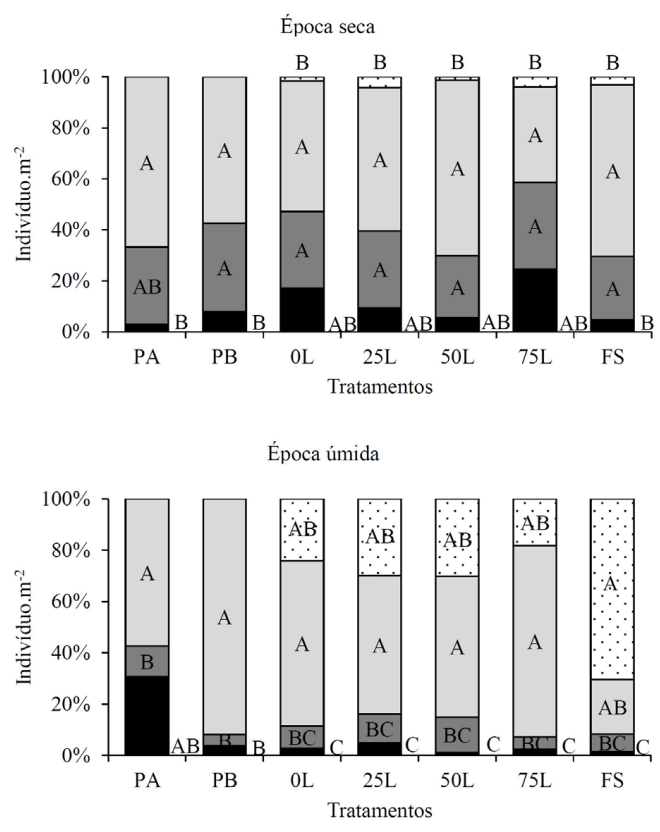

보 Serapilheira $\square 0-10 \mathrm{~cm} \quad \square 10-20 \mathrm{~cm} \square 20-30 \mathrm{~cm}$

Figura 1. Distribuição vertical da macrofauna edáfica (serapilheira, 0-10 cm, 10-20 cm, 20-30 cm) para os tratamentos pasto A (PA), pasto B (PB), 0L, 25L, 50L, 75L e floresta secundária (FS) nas épocas seca (agosto/2007) e chuvosa (fevereiro/2008). Médias em cada área, seguidas por letras diferentes, apresentam diferença $(\mathrm{p}<0,05)$ pelo teste não paramétrico de Kruskal-Wallis.

Figure 1. Vertical distribution of soil macrofauna (litter, $0-10 \mathrm{~cm}, 10-20 \mathrm{~cm}, 20-30 \mathrm{~cm}$ ) for treatments pasture A (PA), Pasture B (PB), 0 L, 25 L, 50 L, 75 L and secondary forest (FS) in dry (August/07) and rainy (February/2008) times. Ratings in each area, followed by different letters show differences $(p<0.05)$ by nonparametric Kruskal-Wallis.

A Figura 2 mostra a distribuição dos grupos que proporcionaram diferenças entre as camadas superficiais (serapilheira e 0-10 cm) nas épocas estudadas.

Além do grande domínio do grupo formicidae em todos os tratamentos, como discutido anteriormente, na profundidade de $0-10 \mathrm{~cm}$ do solo, durante a época chuvosa, ocorreu um grande número de indivíduos do grupo oligochaeta em todos os tratamentos, destacando-se por não terem ocorrido da mesma forma no período anterior. As minhocas tendem a ser mais exigentes em termos de umidade, temperatura e matéria orgânica do solo, sendo observado em algumas espécies um comportamento migratório no perfil do
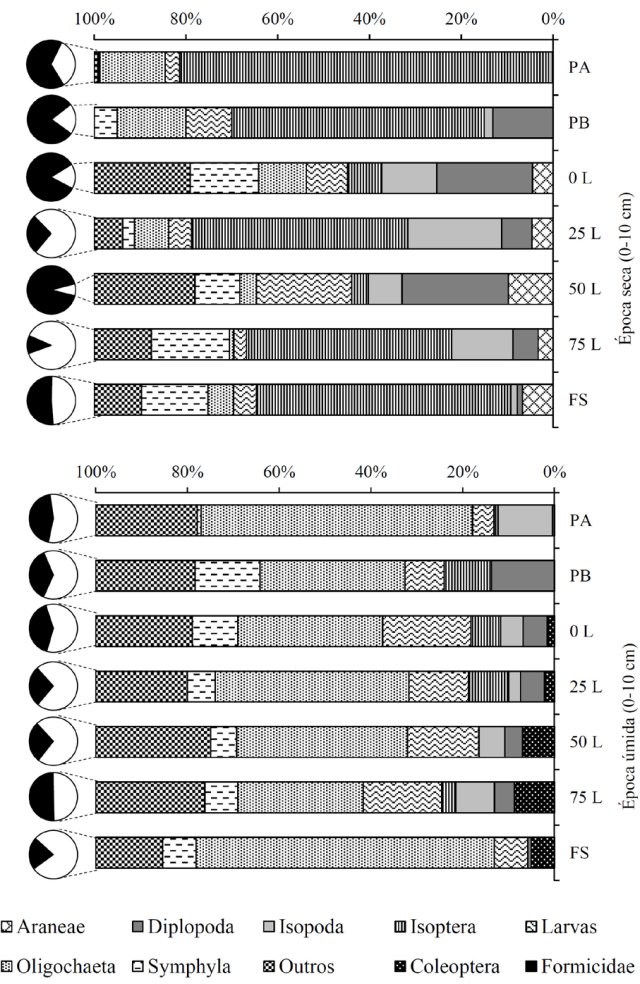

Figura 2. Composição relativa da macrofauna do solo superficial $(0-10 \mathrm{~cm})$ expressa em porcentagem do total de indivíduos. $\mathrm{m}^{-2}$ em cada uma das áreas. PA - pasto A; PB - pasto B; $0 \mathrm{~L}-0 \%$ de leguminosas; $25 \mathrm{~L}-25 \%$ de leguminosas; $50 \mathrm{~L}$ - 50\% de leguminosas; 75L - 75\% de leguminosas; FS - Floresta secundária.

Figure 2. Macrofauna composition of surface soil $(0-10 \mathrm{~cm})$ expressed as a percentage of total individuals $\mathrm{m}^{-2}$ in each of the areas. PA - Pasture A, PB - Pasture B; 0L - $0 \%$ of legumes; $25 \mathrm{~L}-25 \%$ of legumes; $50 \mathrm{~L}-50 \%$ of legumes; 75L - 75\% of legumes, FS - secundary forest.

solo, em função de variações na temperatura e umidade (Chan, 2001; Dias et al., 2007).

O grupo isoptera apresentou densidades mais expressivas no período seco comparativamente ao período chuvoso. Os cupins apresentam a incrível habilidade de permanecerem ativos em ambientes severos, ou durante estações secas, durante as quais a maior parte dos artrópodes diminui sua atividade, pois conseguem manter a umidade nos seus ninhos (Jouquet et al., 2011). Enquanto isso, os demais grupos foram mais abundantes, de um modo geral, durante o verão, na época úmida. Mudanças na umidade do solo, por afetarem indivíduos decompositores, resultam em mudanças na composição da comunidade 

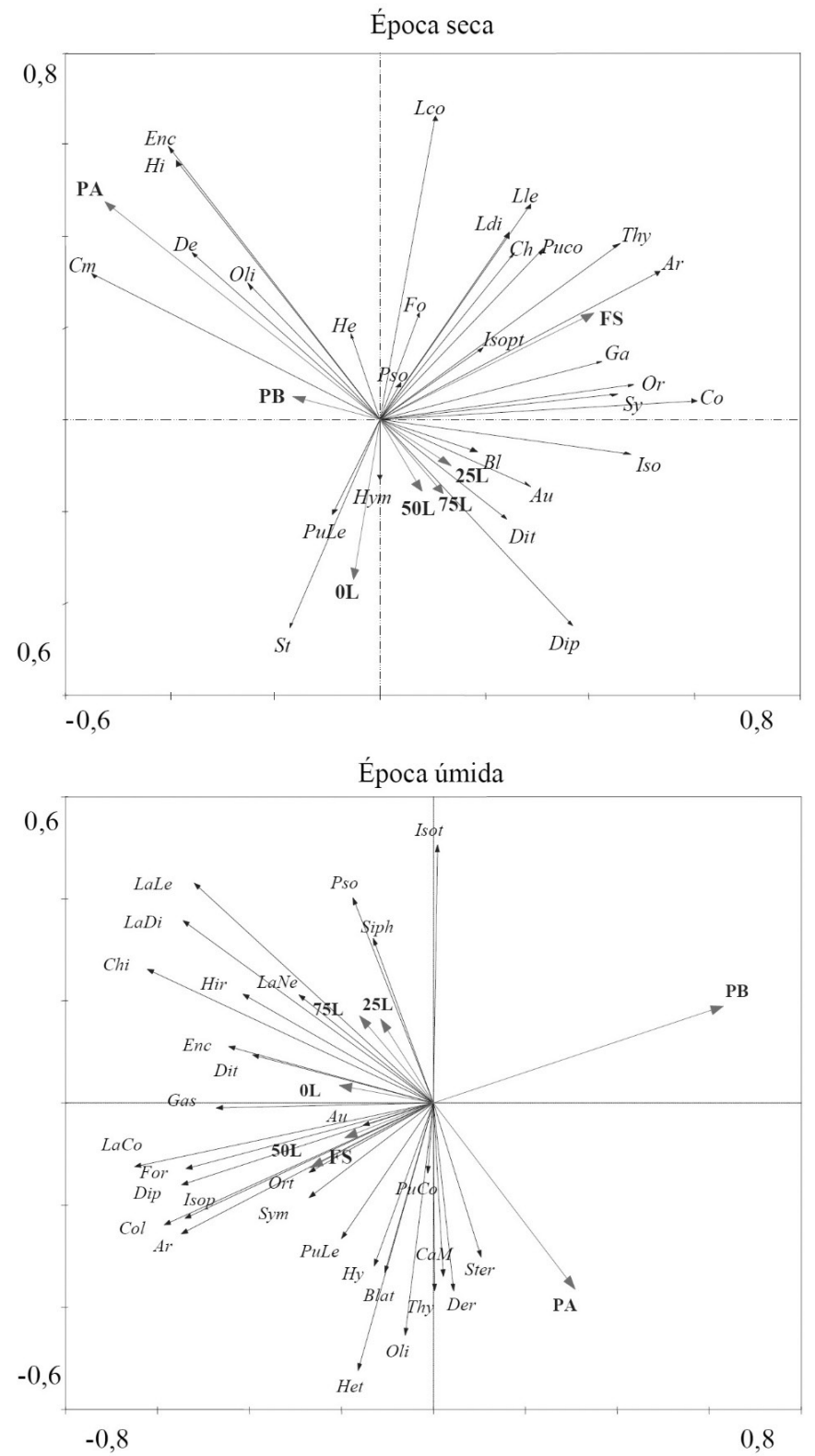

Figura 3. Análise dos componentes principais e atributos da macrofauna do solo nas diferentes áreas avaliadas na época seca e úmida. PA - pasto A; PB - pasto B; 0L - 0\% de leguminosas; 25L - 25\% de leguminosas; 50L - 50\% de leguminosas; 75L - 75\% de leguminosas; FS - Floresta secundária; Ar - Araneae; Au - Auchenorryncha; Bl - Blattodea; Ch - Chilopoda; Cm - Casulo de minhoca; Co - Coleoptera; De - Dermaptera; Dip - Diplopoda; Dit - Diptera; Enc - Enchytraeidae; Fo - Formicidae; Ga - Gastropoda; He - Heteroptera; Hi - Hirudinea; Hym - Hymenoptera; Iso - Isopoda; Isop - Isoptera; Oli - Oligocheta; Or - Orthoptera; Lco - Larva de coleoptera; Ldi - Larva de diptera; Lle - Larva le Lepdoptera; Pso - Psocoptera; Puco - Pulpa de coleoptera; PuLe - Pulpa de lepdoptera; St - Sternorryncha; Sy - Symphila; Thy - Thysanura.

Figure 3. Principal components analysis of the attributes of soil macrofauna in different evaluated areas of dry and wet season. PA - Pasture A, PB - Pasture B; 0L - 0\% of legumes; 25L - 25\% of legumes; 50L - 50\% of legumes; 75L - 75\% of legumes, FS - secundary forest, Ar - Araneae, Au - Auchenorryncha, Bl - Blattodea, Ch - Chilopoda; Cm - Cocoon worm, Co - Coleoptera; De - Dermaptera, Dip - Diplopoda; Dit - Diptera; Enc - Enchytraeidae; Fo - Formicidae; Ga - Gastropoda, He - Heteroptera, Hi - Hirudinea, Hym - Hymenoptera; Iso - Isopoda; Isop - Isoptera; Oli - Oligocheta; Or - Orthoptera; Lco - Coleoptera worm; Ldi - Diptera worm; Lle - Lepdoptera worm; Pso - Psocoptera; Puco - Coleoptera Pulp; skip - Lepdoptera Pulp; St. - Sternorryncha; Sy - Symphila; Thy - Thysanura. 
e no papel funcional da comunidade como um todo (Collison et al., 2013).

A análise dos componentes principais (Figura 3) mostrou que na época seca a maior diversidade da fauna do solo está relacionada aos ambientes em nível maior na escala de sucessão vegetal, ou seja, à floresta secundária e às áreas revegetadas com leguminosas fixadoras de nitrogênio. $\mathrm{O}$ tratamento $\mathrm{OL}$, no qual não houve plantio desse grupo, comportou-se com maior similaridade com as áreas de pasto. Já no período chuvoso essa tendência encontrada foi ainda mais acentuada, grupos da fauna relacionaram-se acentuadamente aos tratamentos que possuíam cobertura vegetal mais rica e diversa.

\section{CONCLUSÕES}

O processo de revegetação associado ao período amostral influenciou de forma positiva a dinâmica dos organismos da macrofauna do solo, proporcionando uma comunidade mais diversa e melhor estruturada, embora não mais abundante.

\section{STATUS DA SUBMISSÃO}

Recebido: 03 abr., 2014

Aceito: 15 nov., 2016

\section{AUTOR(ES) PARA CORRESPONDÊNCIA}

\section{Miriam de Oliveira Bianchi}

Departamento de Solos, Universidade Federal Rural do Rio de Janeiro - UFRRJ, Rodovia BR 465, s/n, Zona Rural, Km 7, CEP 23897-970, Seropédica, RJ, Brasil e-mail: miriambianchii@yahoo.com.br

\section{REFERENNCIAS}

Anderson JM, Ingram JSI. Tropical Soil Biological and Fertility: a handbook of methods. 2nd ed. Wallingford: CAB International; 1993.

Ashford OS, Foster WA, Turner BL, Sayer EJ, Sutcliffe L, Tanner EVJ. Litter manipulation and the soil arthropod community in a lowland tropical rainforest. Soil Biology \& Biochemistry 2013; 62: 5-12. http://dx.doi.org/10.1016/j. soilbio.2013.03.001.

Binkley D, Fisher RF. Ecology and management of forest soils. 4th ed. Oxford: Wiley-Blackwell; 2013.
Chan KY. An overview of some tillage impacts on earthworm population abundance and diversity - implications for functioning in soils. Soil \& Tillage Research 2001; 57(4): 179-191. http://dx.doi.org/10.1016/S0167-1987(00)00173-2.

Collison EJ, Riutta T, Slade EM. Macrofauna assemblage composition and soil moisture interact to affect soil ecosystem functions. Acta Oecologica 2013; 47: 30-36. http://dx.doi.org/10.1016/j.actao.2012.12.002.

Dias PF, Souto SM, Correia MEF, Rocha GP, Moreira JF, Rodrigues KM et al. Árvores fixadoras de nitrogênio e macrofauna do solo em pastagem de híbrido de Digitaria. Pesquisa Agropecuária Brasileira 2006; 41(6): 1015-1021. http://dx.doi.org/10.1590/S0100-204X2006000600018.

Dias PF, Souto SM, Correia MEF, Rodrigues KM, Franco AA. Efeito de leguminosas arbóreas sobre a macrofauna do solo em pastagem de Brachiaria brizantha cv. Marandu. Pesquisa Agropecuária Tropical 2007; 37(1): 38-44.

Dindal D. Soil biology guide. New York: John Wiley and Sons; 1990.

Duarte EMG, Cardoso IM, Stijnen T, Mendonça MAFC, Coelho MS, Cantarutti RB et al. Decomposition and nutrient release in leaves of Atlantic Rainforest tree species used in agroforestry systems. Agroforestry Systems 2013; 87(4): 835-847. http://dx.doi.org/10.1007/s10457-013-9600-6.

Gei MG, Powers JS. Do legumes and non-legumes tree species affect soil properties in unmanaged forests and plantations in Costa Rican dry forests? Soil Biology \& Biochemistry 2013; 57:264-272. http://dx.doi.org/10.1016/j. soilbio.2012.09.013

Instituto Brasileiro de Geografia e Estatística - IBGE. Manual técnico da vegetação brasileira. 2. ed. Rio de Janeiro: IBGE; 2012.

Jeffery S, Gardi C, Jones A, Montanarella L, Marmo L, Miko $\mathrm{L}$ et al. European atlas of soil biodiversity. Luxembourg: Publications Office of the European Union; 2010.

Jouquet P, Traoré S, Choosai C, Hartmann C, Bignell D. Influence of termites on ecosystem functioning. Ecosystem services provided by termites. European Journal of Soil Biology 2011; 47(4): 215-222. http://dx.doi.org/10.1016/j. ejsobi.2011.05.005.

Lavelle P. Faunal activities and soil processes: adaptative strategies that determine ecosystem function. Advances in Ecological Research 1997; 27: 93-132. http://dx.doi. org/10.1016/S0065-2504(08)60007-0.

Lepsch IF. 19 lições de pedologia. São Paulo: Oficina de textos; 2011.

Manhães CMC, Gama-Rodrigues EF, Moço MKS, GamaRodrigues AC. Meso- and macrofauna in the soil and litter of leguminous trees in a degraded pasture in Brazil. Agroforestry Systems 2013; 87(5): 993-1004. http://dx.doi. org/10.1007/s10457-013-9614-0.

Moraes LFD, Assumpção JM, Pereira TS, Luchiari C. Manual técnico para a restauração de áreas degradadas 
no estado do Rio de Janeiro. Rio de Janeiro: Instituto de Pesquisas, Jardim Botânico do Rio de Janeiro; 2013.

Riutta T, Slade EM, Bebber DP, Taylor ME, Malhi Y, Riordan $P$ et al. Experimental evidence for the interaction effects of forest edge, moisture and soil macrofauna on leaf litter decomposition. Soil Biology \& Biochemistry 2012; 49: 124-131. http://dx.doi.org/10.1016/j.soilbio.2012.02.028.

Rousseau GX, Deheuvels O, Arias LR, Somarriba E. Indication soil quality in cacao-based agroforestry systems and old-growth forest: the potential of soil macrofauna assemblage. Ecological Indicators 2012; 23: 535-543. http:// dx.doi.org/10.1016/j.ecolind.2012.05.008.

Spolidoro MLCV. Composição e estrutura de um trecho de floresta no médio Paraíba do Sul, RJ [dissertação]. Seropédica: Instituto de Florestas, Universidade Federal Rural do Rio de Janeiro; 2001.

Vasconcellos RLF, Segat JC, Bonfim JA, Baretta D, Cardoso EJBN. Soil macrofauna as an indicator of soil quality in a undisturbed ripariam forest and recovering sites of different ages. European Journal of Soil Biology 2013; 58: 105-112. http://dx.doi.org/10.1016/j.ejsobi.2013.07.001. 